\title{
AVALANCHES OF MAGNETIC RECONNECTION
}

\author{
EDWARD T. LU \\ Institute for Astronomy, 2680 Woodlawn Drive, Honolulu, HI 96822, USA
}

\begin{abstract}
Active region coronal magnetic fields are expected to be in a twisted tangled state due to photospheric convective motions. These motions can drive the magnetic field to a statistically steady state where energy is released impulsively ( $\mathrm{Lu}$ and Hamilton 1991). These relaxation events in the magnetic field can be interpreted as avalanches of many small reconnection events. We argue that the frequency distribution of these magnetic reconnection avalanches must be a power law. Furthermore, we calculate the expected distributions in a simple model of magnetic energy release events in a 3 -dimensional complex magnetized plasma, and compare these to the distributions of solar flares. These distributions are found to match the observed power law distributions of solar flare energies, peak fluxes, and durations. This model implies that the energy release process is fundamentally the same for flares of all sizes. Observational predictions of this model are discussed.
\end{abstract}

Any complete theory of solar flares (or of any astronomical objects) must address not only the question of the physics underlying individual events, but also the question of the overall distributions of the observed events. While there has been much work devoted to studying the physics of individual solar flares, little attention has been paid to understanding the frequency distributions of solar flares. Solar flares are observed to have a very large dynamic range in both energy and duration. The total energy release ranges from less than $10^{27}$ ergs in the smallest observable flares to of order $10^{33} \mathrm{ergs}$, while the durations range from less than of order one second to several thousand seconds. These are remarkable ranges in energy and duration, yet the distribution of solar flare peak luminosities is observed to be a featureless power law over a dynamic range of more than four orders of magnitude, extending down to the limits of observation (Dennis 1985). In addition to having a large dynamic range in energy and duration, solar flares are observed to have structure on length scales from more than $10^{9} \mathrm{~cm}$ to well below $10^{8} \mathrm{~cm}$. Imaging observations show that the geometry of flaring regions is usually not simple, and often contains structure down to the instrument resolution. Any complete theory of solar flares must be able to account not only for the observed emissions and morphology of flares, but for the reasons behind this broad distribution of flares. In this paper, we briefly describe an idea ( $\mathrm{Lu} \&$ Hamilton 1991, hereafter LH91) that can quantitatively account for the observed frequency distributions of solar flares. In LH91, we suggest that the dynamics of a highly complicated magnetized plasma can be understood using a statistical approach, and that the distributions of solar flares 
arise naturally from the physics of the energy release process.

The problem of understanding the energy release process in such a complicated configuration is extremely difficult. However, we have suggested that the complexity of the magnetic field structure makes it possible to understand the large scale dynamics of the magnetic field in a statistical manner (LH91). We proposed that the small scale structure in the field leads to a well defined large scale behavior with certain statistical properties, and that the frequency distributions of solar flares arise directly from a few basic properties of the energy release process in the highly complex field of solar active regions. Furthermore, we argue that many of the details of the microscopic processes involved in magnetic reconnection do not affect the large scale behavior of the system. This is reminiscent of the approach used to understand thermodynamic systems, where many useful physical relations among macroscopic quantities are derived without considering the microphysical processes in detail. In a similar way we have argued that much of the large scale behavior of solar active region magnetic fields may be understood without a detailed consideration of all of the microphysics.

To illustrate this, we have constructed a simple model of energy release in active regions which has many of the features of active region magnetic fields, yet whose dynamical rules are simple enough that the detailed evolution of the system can be calculated. We consider the behavior of a vector field defined on a 3 dimensional grid. We define a local instability criterion, and drive the system by randomly adding small vectors to random locations on the grid. When an instability occurs at some point, the field readjusts locally to make it again stable at that point. However, this process also changes the field at neighboring points which may then cause neighboring locations to become unstable. Thus the energy release process can spread throughout the system. In this model flares are avalanches of many energy release events, with larger flares simply being composed of a greater number of elementary events. When randomly driven the field eventually reaches a statistically steady state where the distributions of flares do not change. This state is completely independent of the initial configuration of the field, so that we can expect that the active region magnetic fields might have similar statistical properties. Furthermore, in this steady state, the distributions of the energy release events as a function of duration, total energy released, and peak luminosity are fairly insensitive to the instability criterion and the way in which the field readjusts to a local instability. In fact the distributions of avalanche properties in this model quantitatively match the observed frequency distributions of solar flares (see Fig. 1). Thus it may be possible to understand many of the properties of flares without a detailed consideration of all of the microphysics. For instance, the frequency distributions of flares are found to arise naturally from only a few basic properties of local magnetic reconnection instabilities. A much more complete description of the model and its justification are given in Lu and Hamilton $(1991,1992)$.

\section{RFFERENCES}

Bromund, K. McTiernan, J., and Kane, S. in preparation

Dennis, B. 1985, Solar Phys., 100, 465 
Lu, E.T., and Hamilton, R.J. 1991, ApJ, 380, L89

Lu, E.T., and Hamilton, R.J. 1992, ApJ, submitted.
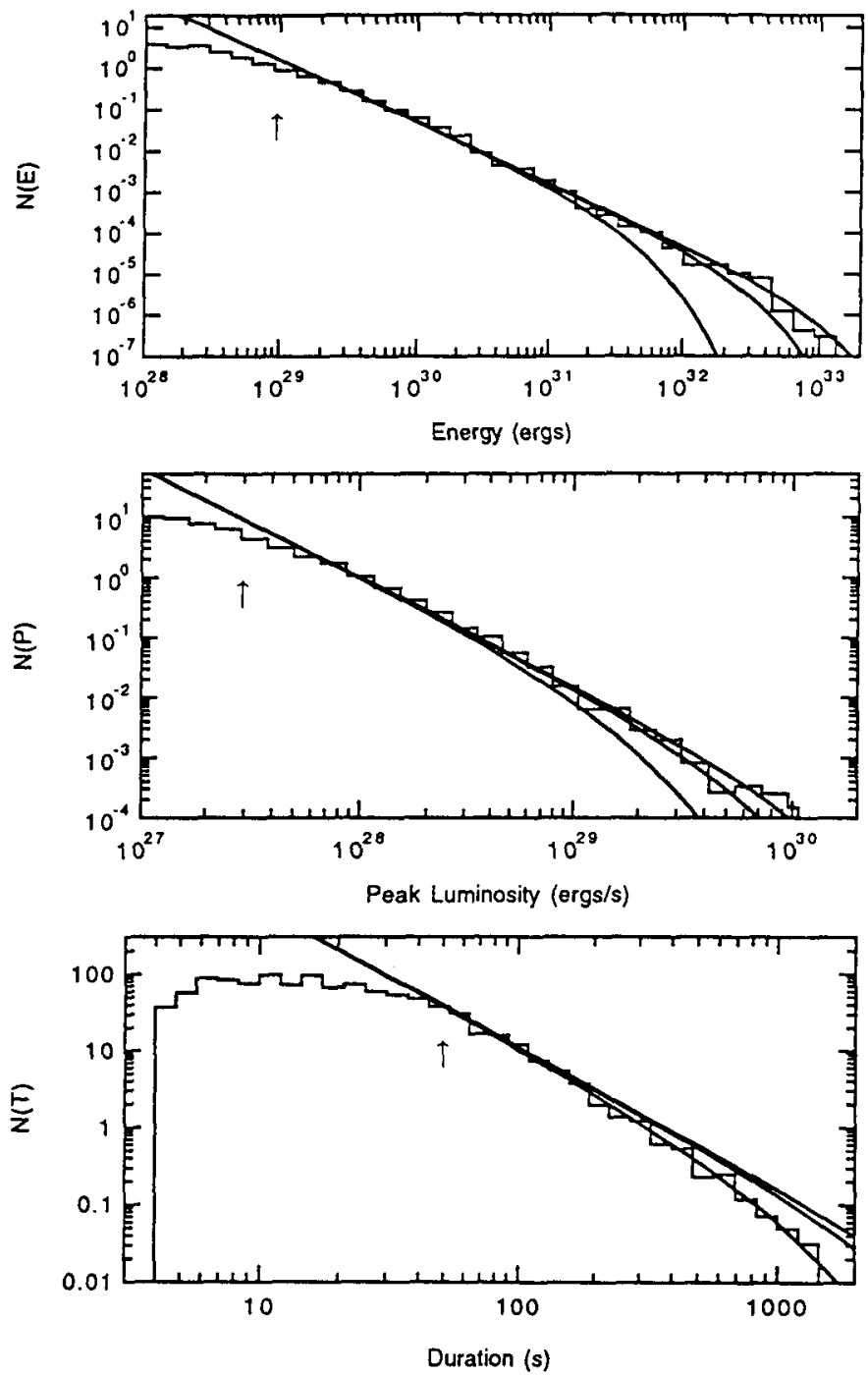

Figure -Comparison between the frequency distributions of flares observed by ISEE-3/ICE (Bromund, McTiernan, \& Kane 1992) (histogram) as a function of flare energy, peak luminosity, and duration with the theoretically predicted distributions (solid lines) for active region sizes $L_{a}=3 \times 10^{9} \mathrm{~cm}, 5 \times 10^{9} \mathrm{~cm}$, and $7 \times 10^{\circ} \mathrm{cm}$. The observed distributions are incomplete below the arrows. 